\title{
PCM-trace: Scalable Synaptic Eligibility Traces with Resistivity Drift of Phase-Change Materials
}

\author{
Yiğit Demirağ ${ }^{1}{ }^{*}$, Filippo Moro ${ }^{2}$, Thomas Dalgaty ${ }^{3}$, Gabriele Navarro ${ }^{2}$, Charlotte Frenkel ${ }^{1}$, Giacomo Indiveri ${ }^{1}$, \\ Elisa Vianello ${ }^{2}$, and Melika Payvand ${ }^{1}$ \\ ${ }^{1}$ Institute of Neuroinformatics, University of Zurich and ETH Zurich, Switzerland \\ ${ }^{2}$ CEA-Leti, Grenoble, France, ${ }^{3}$ CEA-List, Nano-INNOV, Université Paris-Saclay, France
}

\begin{abstract}
Dedicated hardware implementations of spiking neural networks that combine the advantages of mixed-signal neuromorphic circuits with those of emerging memory technologies have the potential of enabling ultra-low power pervasive sensory processing. To endow these systems with additional flexibility and the ability to learn to solve specific tasks, it is important to develop appropriate on-chip learning mechanisms. Recently, a new class of three-factor spike-based learning rules have been proposed that can solve the temporal credit assignment problem and approximate the error back-propagation algorithm on complex tasks. However, the efficient implementation of these rules on hybrid CMOS/memristive architectures is still an open challenge. Here we present a new neuromorphic building block, called PCM-trace, which exploits the drift behavior of phasechange materials to implement long lasting eligibility traces, a critical ingredient of three-factor learning rules. We demonstrate how the proposed approach improves the area efficiency by $>10 \times$ compared to existing solutions and demonstrates a technologically plausible learning algorithm supported by experimental data from device measurements.
\end{abstract}

\section{INTRODUCTION}

Neuromorphic engineering uses electronic analog circuit elements to implement compact and energy-efficient intelligent cognitive systems [1]-[4]. Leveraging substrate's physics to emulate biophysical dynamics is a strong incentive toward ultra-low power and real-time implementations of neural networks using mixed-signal memristive event-based neuromorphic circuits $[5]-[8]$. The majority of these systems are currently deployed in edge-computing applications only in inference mode, in which the network parameters are fixed. However, learning in edge computing can have many advantages, as it enables adaptation to changing input statistics, reduced network congestion, and increased privacy. Indeed, there have been multiple efforts implementing Spike-Timing Dependent Plasticity (STDP)-variants and Hebbian learning using neuromorphic processors [9]-[11]. These methods control Long Term Depression (LTD) or Long Term Potentiation (LTP) by specific local features of pre- and post-synaptic activities. However, local learning rules themselves do not provide any guarantee that network performance will improve in multi-layer or recurrent networks. Local error-driven approaches, e.g., the Delta Rule, aim to solve this problem but fail to assign credit for neurons that are multiple synapses away from the network output [12], [13]. On the other hand, it has been recently shown that by using external thirdfactor neuromodulatory signals (e.g., reward or prediction error in reinforcement learning, teaching signal in supervised learning), this can be achieved in hierarchical networks [14],
[15]. However, there needs to be a mechanism for synapses to remember their past activities for long periods of time, until the reward event or teacher signal is presented. In the brain, these signals are believed to be implemented by calcium ions, or CAMKII enzymes in the synaptic spine [16] and are called eligibility traces. In machine learning, algorithmic top-down analysis of the gradient descent demonstrated how local eligibility traces at synapses allow networks to reach performances comparable to error back-propagation algorithm on complex tasks [17]-[19]. Examples of neuromorphic platforms that implement these types of eligibility traces in spiking neural networks already exist [20]-[22]. However, learning in these platforms is only supported through the use of von-Neumann processors, either shared with the computation of network dynamics [21] or a dedicated core [20], [22]. Relying on numerical integration, these platforms do not leverage the physics of their computing substrate and are not free from the von-Neumann bottleneck problem [23], [24]. On the other hand, mixed-signal analog/digital neuromorphic circuits allow the use of in-memory computing that directly emulates the desired neural and synaptic dynamics using the physics of analog elements [25]-[27]. However, even though progress has been made in extending the duration of synaptic traces using advanced Fully-Depleted Silicon on Insulator (FDSOI) technologies [28], implementing tens-of-secondslong time constants solely based on Complementary MetalOxide-Semiconductor (CMOS) is not scalable, as it requires the use of large capacitors. In this paper, we present a novel approach to exploit the drift behavior of Phase Change Memory (PCM) devices to intrinsically perform Eligibility Trace (ET) computation over behavioral timescales. We present the PCMtrace building block as a hybrid memristive-CMOS circuit solution that can lead to record-low area requirements per synapse. To the best of our knowledge, this is the first work that uses a memristive device not only to store the weight of synapses, but also to keep track of synaptic eligibility to interact with a third factor toward scalable next-generation onchip learning.

\section{Eligibility Traces}

The ET can be described as a decaying synaptic variable as in Eq. (11). The value of the ET at the synapse between presynaptic neuron $j$ and post-synaptic neuron $i$ can be controlled as a usually linear function, $f_{j}$, of the pre-synaptic activity $x_{j}$, and potentially non-linear function $g_{i}$ of the post-synaptic activity $x_{i}$ such that

$$
e_{i j}^{t+\Delta t}=\alpha e_{i j}^{t}+\eta f_{j}\left(x_{j}^{t}\right) g_{i}\left(x_{i}^{t}\right)
$$




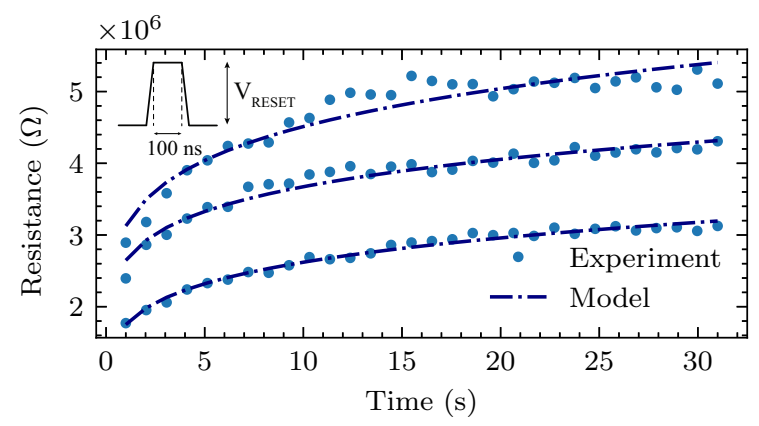

Fig. 1: Experimental (dots), and simulated (dashed lines) resistance drift characteristics at constant room temperature.

where $\eta$ is a constant and $\alpha=e^{-\Delta t / \tau_{m}}$ is the decay rate of ET, $\tau_{m}$ is decay time-constant up to tens of seconds in behavioural time-scales and $\Delta t$ is discrete time-step [14]. The $e_{i j}$ acts as a temporal correlation detector between pre-synaptic $f_{j}\left(x_{j}\right)$ and post-synaptic $g_{i}\left(x_{i}\right)$ functions. The instantaneous correlation between $f_{j}$ and $g_{i}$ is defined as synaptic tagging, which is accumulated by $e_{i j}$ to keep track of past correlations. The $f$ and $g$ functions are determined by the chosen synaptic learning rule. For example, $f_{j}\left(x_{j}\right)$ is the low-pass filtered presynaptic events in e-prop and BDSP [17], [29], and $g_{i}\left(x_{i}\right)$ is a non-linear function of the post-synaptic state for e-prop and SuperSpike [18].

\section{PCM MEASUREMENTS}

Temporal evolution of electrical resistivity is a widelyobserved phenomenon in PCM due to the rearrangements of atoms in the amorphous phase [30]. This behavior is commonly referred to as structural relaxation or drift. To start the drift, a strong RESET pulse is applied to induce a crystalline to amorphous phase transition where the PCM is melted and quenched. The low-ordered and highly-stressed amorphous state then evolves to a more energetically favorable glass state within tens of seconds [31].

At constant ambient temperature, the resistivity follows

$$
R(t)=R\left(t_{0}\right)\left(\frac{t}{t_{0}}\right)^{\nu},
$$

where $R\left(t_{0}\right)$ is the resistance measured at time $t_{0}$ and $\nu$ is the drift coefficient. It has been experimentally verified by many groups that Eq. (2) can successfully capture the drift dynamics [31]-[33], from microseconds to hours range [34].

We integrated $\mathrm{Ge}_{2} \mathrm{Sb}_{2} \mathrm{Te}_{5}$-based PCM in state-of-the-art PCM heater-based devices fabricated in the Back-End-Of-Line (BEOL) based on $130 \mathrm{~nm}$ CMOS technology. The PCM thickness is $50 \mathrm{~nm}$ with the bottom size of $60 \mathrm{~nm} \times 60 \mathrm{~nm}$. Drift measurements were performed on three devices to monitor the temporal evolution of the resistance in the High-Resistive State (HRS) state, particularly confirming the model in Eq. (2). The test was conducted by first resetting all the cells by applying a RESET pulse to the heater, which has a width of $100 \mathrm{~ns}$ with $5 \mathrm{~ns}$ rising and falling times, and a peak voltage of $1.85 \mathrm{~V}$. Then, an additional programming pulse is used to bring the devices to different initial conditions, corresponding

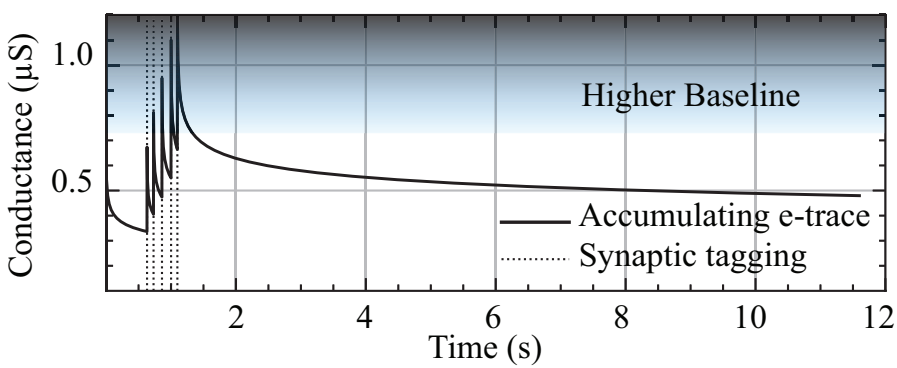

Fig. 2: Accumulating ET using PCM-trace drift model (Eq. 3). After resetting the PCM-trace device at $t=0,5$ random synaptic tags are applied to the synapse, implemented by a gradual SET for each tag that results in $50 \%$ increase in the conductivity. The device can keep the ET for more than $10 \mathrm{~s}$.

to $R(t=1 \mathrm{~s})=[1.77 \mathrm{M} \Omega, 2.39 \mathrm{M} \Omega, 2.89 \mathrm{M} \Omega]$. The low-field device resistances are measured every $1 \mathrm{~s}$ for $30 \mathrm{~s}$ by applying a READ pulse which has the same timing of the RESET pulse but a peak voltage of $0.05 \mathrm{~V}$.

\section{PCM-TRACE}

PCM-trace is a novel method to implement seconds-long ET for the synapse using the drift feature of PCM. By writing Eq. (2) as a difference equation of the conductance, we can show that the temporal evolution of the conductance has decay characteristics similar to Eq. (1) such that $G_{i j}^{t+\Delta t}=\left(\frac{t-t_{p}}{t-t_{p}+\Delta t}\right)^{\nu} G_{i j}^{t}$, where $G_{i j}^{t_{0}}=1 / R_{i j}^{t_{0}}$, and $t_{p}$ is the last programming time as drift re-initializes with every gradual SET [35], [36]. The main difference is that the rate of change in PCM resistivity is a function of time; nevertheless, its time constant is comparable for behavioral time-scales as $\tau_{P C M}=-\Delta t / \log \left((t /(t+\Delta t))^{\nu}\right)$ is on the order of tens of seconds [37]. Therefore, the PCM-trace dynamics can emulate the ET of the synapse as follows:

$$
G_{i j}^{t+\Delta t}=\left(\frac{t-t_{p}}{t-t_{p}+\Delta t}\right)^{\nu} G_{i j}^{t}+\eta f_{j}\left(x_{j}^{t}\right) g_{i}\left(x_{i}^{t}\right)
$$

In the PCM-trace method (Eq. 3), the accumulating term on the ET is implemented by applying a gradual SET to the PCM device whenever the synapse is tagged. To maximize the number of accumulations a PCM device can handle without getting stuck in the Low-Resistive State (LRS) regime, some operational conditions need to be satisfied. We initialize the device to HRS by applying a strong RESET pulse, and wait for an initialization time $t_{\text {init }}$ of at least $250 \mathrm{~ms}$ for the device resistance to increase. If $t_{\text {init }}$ is too short, the device conductance would still be too high to be able to accumulate enough tags; and if it is too long, the decay will be weaker (see Eq. 2). Initialization time can be modulated to reach the desired drift speed depending on the material choice and the application. After the initialization time, whenever the synapse is tagged, a single gradual SET (with an amplitude of $100 \mu \mathrm{A}$ and a pulse width of $100 \mathrm{~ns}$ with $5 \mathrm{~ns}$ rising and falling times) is applied. To make sure that the device stays in the HRS, a read-verify-set scheme can be used. Finally, the value of the ET can be measured after seconds by reading the conductance of the device (see Fig. 2). 


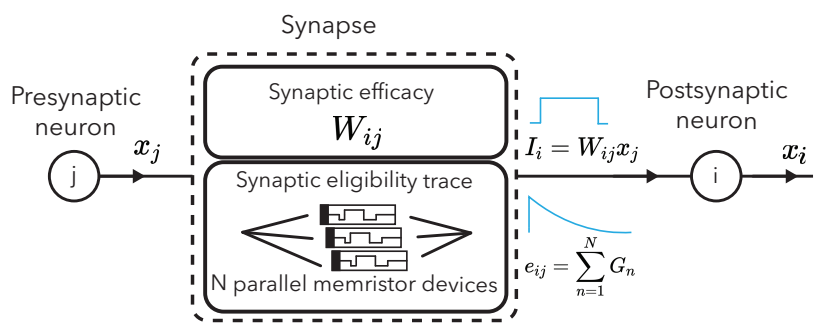

Fig. 3: Multi PCM-trace concept. Each synapse has a weight and a PCM-trace block where multiple parallel PCM devices keep the ET of the synapse with their natural drift behavior. The postsynaptic neuron receives the sum of product of the pre-synaptic activity and the weight block. In parallel, the PCM-trace block calculates the ET as a function of pre- and post-synaptic activities (Eq. 3), to be used in the weight update.

\section{A. Multi PCM-trace}

The number of gradual SET pulses applied to a single PCMtrace device is limited, because each pulse partially increases the device conductivity and eventually move the device toward its LRS $(<2 \mathrm{M} \Omega)$, where the drift converges to a higher baseline level. This problem can be solved by storing the synaptic ET distributed across multiple PCM devices, as in Fig. 3 By successively routing the tags to multiple PCM devices, the number of gradual SET pulses to be applied per single device is significantly reduced. Fig. 4 demonstrates the increase of effective dynamic range (number of updates to ET without getting stuck in the LRS) using multiple PCM devices.

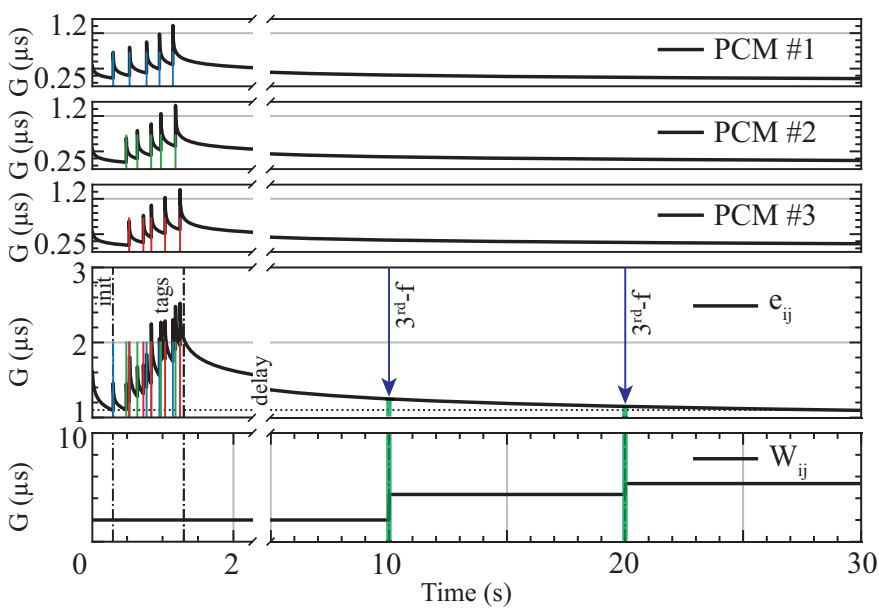

Fig. 4: Accumulating ET using multi-PCM configuration. Synapse receives 15 tags between $300 \mathrm{~ms}$ to $1300 \mathrm{~ms}$ which are routed to three different devices shown in the top three plots. The effective ET is calculated by applying a READ pulse to the parallel PCM devices. The initialization duration and synaptic activity period are shown with dashed lines in the bottom plot. The synaptic efficacy $W_{i j}$ is modified depending on the state of ET once the third-factor signal arrives.

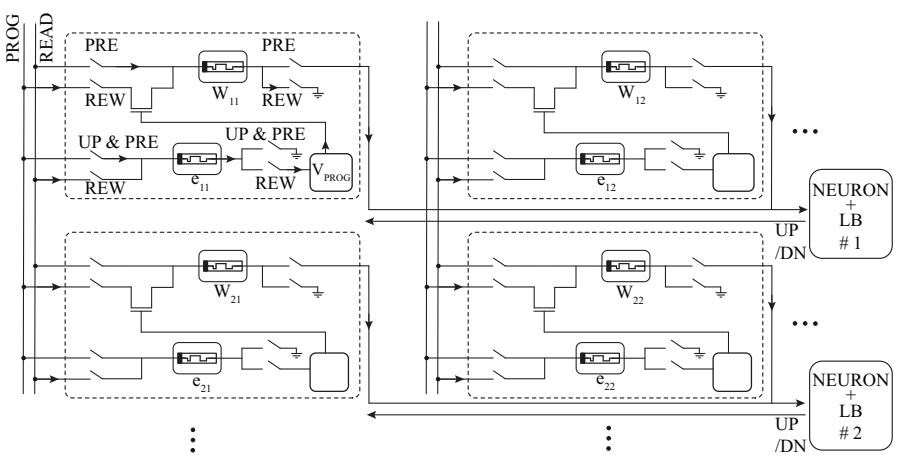

Fig. 5: PCM-trace-based neuromorphic architecture for threefactor learning. Only positive ET $\left(e_{i j}^{+}\right)$and $W_{i j}^{+}$are shown.

\section{Circuit And ARChitecture}

\section{A. PCM-trace Architecture}

An example in-memory event-based neuromorphic architecture is shown in Fig. 5, where the PCM-trace is employed to enable three-factor learning on behavioral time scales.

Synapse: Each synapse includes a weight block $W_{i j}$ in which two PCM devices are used in differential configuration to represent positive and negative weights [38]. The effective synaptic weight is calculated as the difference of these two conductance values, i.e., $W_{i j}=W_{i j}^{+}-W_{i j}^{-}$. Also, each synapse has a PCM-trace block $e_{i j}$ that keeps the ET. Inside the PCM-trace block, there are two PCM devices, keeping track of the positive and negative correlation between pre and post-synaptic neurons. On the onset of the pre-synaptic input spike, $P R E_{j}$, (i) $W_{i j}$ is read, and the current is integrated by the post-synaptic neuron $i$; (ii) Based on the $U P / D N$ signal from the learning block (LB), a gradual SET programming current is applied to positive/negative PCM-trace devices.

Neuron with Learning Block (LB): The LB estimates the pre-post synaptic neuron correlation using the Spike Driven Synaptic Plasticity (SDSP) rule [39]. At the time of the pre-synaptic spike, the post-synaptic membrane variable is compared against a threshold, above (below) which an $U P$ $(D N)$ signal is generated representing the tag type. On the arrival of the third factor binary reward signal, $R E W$, the state of the ETs devices is read by the $V_{P R O G}$ block (Fig. 6b) which generates a gate voltage that modulates the current that programs the weight devices $W_{i j}$ (see Alg. 1).

\section{B. Circuit simulation}

Fig. 6 describes the block diagram of the LB implementing SDSP rule, which calculates the pre-post neurons' correlation. The membrane variable (described here as a current $I_{m e m}$ since circuits are in current-mode) is compared against a threshold value $I_{t h}$ through a Bump circuit [38], [40]. The output of this block is digitized through a current comparator (in our design chosen as a Winner-Take-All (WTA) block [41]) and generates $U P / D N$ signals if the membrane variable is above/below the threshold $I_{t h}$, and STOP, $S P$, if they are close within the dead zone of the bump circuit [40]. Fig. 6b presents the circuit schematic which reads the PCM-trace and generates $V_{P R O G}$. To read the state of the device, a voltage 


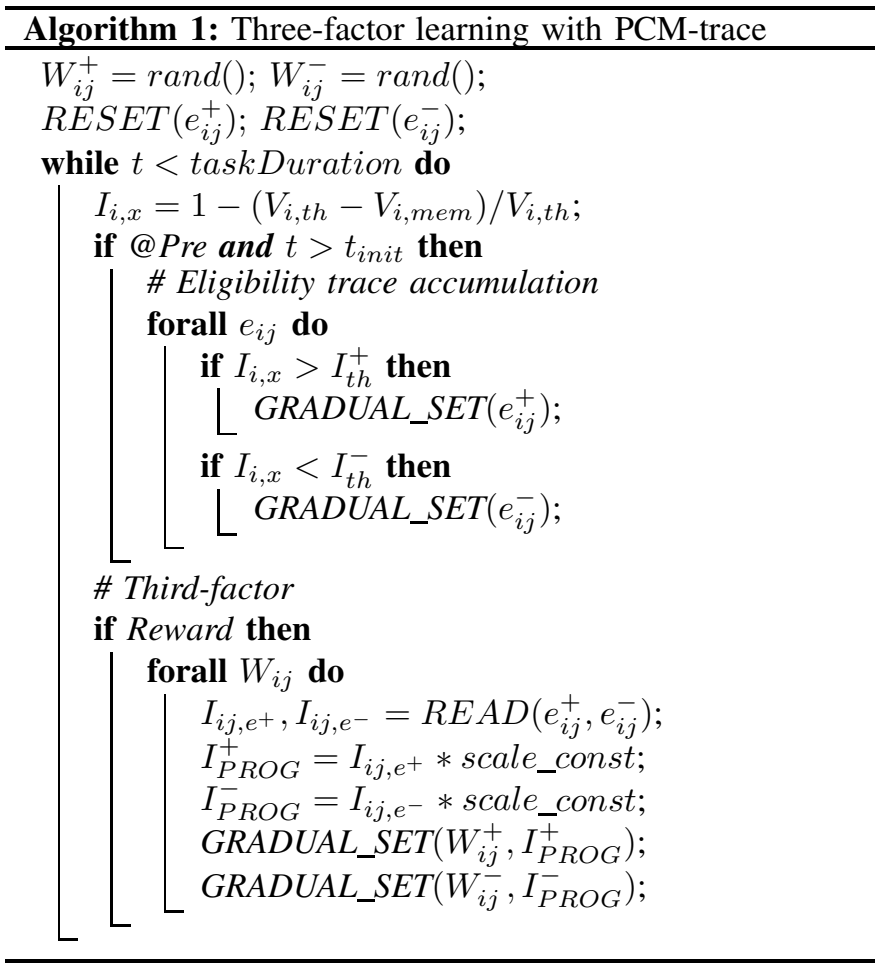

divider is formed between the PCM device and a pseudo resistor, highlighted in green. As the device resistance changes, the input voltage to the differential pair, highlighted in red, changes. This change is amplified by the gain of the diff. pair and the device current is normalized to its tail current giving rise to $I_{P R O G}$ which develops $V_{P R O G}$ through the diodeconnected NMOS transistor. $V_{P R O G}$ is connected to the gate of the transistor in series with the weight PCM (see Fig. 5). Fig. $7 \mathrm{l}$ plots PRE, $I_{m e m}$, the output of the learning block at the time of the PRE, and the gradual SET pulse applied to the device. As shown, the $U P$ signal is asserted when the membrane current is higher than the threshold indicated in red, which causes a gradual SET pulse with $100 \mu \mathrm{A}$ to be applied across the PCM-trace device upon PRE events. Fig $7 \mathrm{~b}$ shows the generated $I_{P R O G}$ as a function of the state of the ET device. The higher the ET device's resistance, the less the accumulated correlation, thus the lower the programming current that should be applied to the weight device. The resistance on the $\mathrm{x}$ axis of the plot matches the measured resistance of PCM devices shown in Fig. 1.

\section{DiscuSSION AND CONCLUSION}

Long-lasting ETs enable the construction of powerful learning mechanisms for solving complex tasks by bridging the synaptic and behavioral time-scales. In this paper, for the first

TABLE I: Area comparison of ET implementation

\begin{tabular}{|l|l|l|l|}
\hline & Area $\left(\mu \mathrm{m}^{2}\right)$ & $\tau(\mathrm{s})$ & Area $/ \tau\left(\mu \mathrm{m}^{2} \mathrm{~s}^{-1}\right)$ \\
\hline CMOS $|\mathbf{2 8}|$ & $20 \times 17$ & 6 & 56.6 \\
\hline PCM [This work] & $12 \times 12$ & $>30$ & $<4.8$ \\
\hline
\end{tabular}

(a)
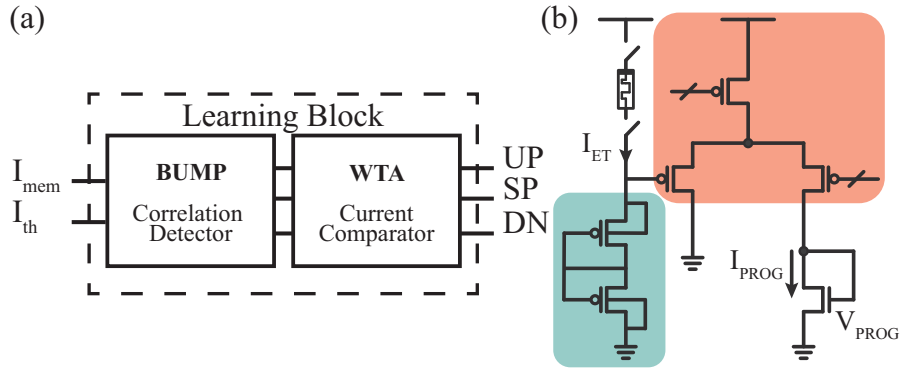

Fig. 6: (a) Learning block diagram generating UP/DN signals as a function of the correlation between pre and post-synaptic activity. (b) $V_{P R O G}$ circuit reading from the ET device through the voltage divider (green) and generating $I_{P R O G}$ through the diff. pair (red) to program the weight device.
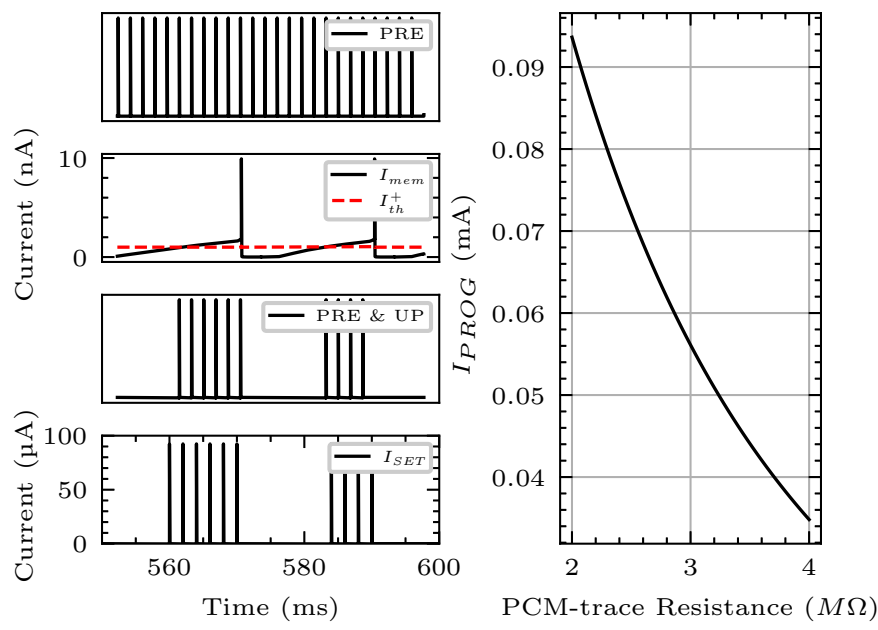

Fig. 7: a) From the top: PRE events, POST membrane current $\left(I_{m e m}\right)$ and learning threshold $\left(I_{t h}\right)$, PRE events only when $I_{m e m}$ is higher than $I_{t h}$, and corresponding gradual SET current pulse applied to PCM-trace. b) Programming current to be applied to the weight PCM as a function of ET state.

time, we proposed to use the drift of PCM devices to implement ETs, and analyzed their feasibility for implementation in existing fabrication technologies.

The implementation of the three-factor learning rules with ETs per synapse requires complex memory structures for keeping track of the ET and the weight. Our proposed approach has clear advantages for scaling. Table I shows a comparison between our PCM synapse and a CMOS-only implementation in $22 \mathrm{~nm}$ FDSOI technology from [28].

PCM is among the most advanced emerging memory technology integrated into the neuromorphic domain [42]. Our approach of using PCM to store both the synaptic weight and the ET requires no additional nano-fabrication methods.

\section{ACKNOWLEDGMENT}

This project has received funding from the European Union's H2020 research and innovation programme under the Marie Skłodowska-Curie grant agreement No 861153, H2020 MeM-Scales project (871371) and ERA-NET CHIST-ERA programme by SNSF (20CH21186999 / 1). 


\section{REFERENCES}

[1] C. Mead, "How we created neuromorphic engineering," Nature Electronics, vol. 3, no. 7, pp. 434-435, 2020.

[2] E. Chicca, F. Stefanini, C. Bartolozzi, and G. Indiveri, "Neuromorphic electronic circuits for building autonomous cognitive systems," Proceedings of the IEEE, vol. 102, no. 9, pp. 1367-1388, 92014.

[3] G. Indiveri and T. Horiuchi, "Frontiers in neuromorphic engineering," Frontiers in Neuroscience, vol. 5, no. 118, pp. 1-2, 2011.

[4] C. Mead, "Neuromorphic electronic systems," Proceedings of the IEEE, vol. 78, no. 10, pp. 1629-36, 1990.

[5] E. Chicca and G. Indiveri, "A recipe for creating ideal hybrid memristive-CMOS neuromorphic processing systems," Applied Physics Letters, vol. 116, no. 12, p. 120501, 2020.

[6] A. Serb, J. Bill, A. Khiat, R. Berdan, R. Legenstein, and T. Prodromakis, "Unsupervised learning in probabilistic neural networks with multi-state metal-oxide memristive synapses," Nature communications, vol. 7, p. 12611, 2016.

[7] Y. Li, Z. Wang, R. Midya, Q. Xia, and J. J. Yang, "Review of memristor devices in neuromorphic computing: materials sciences and device challenges," Journal of Physics D: Applied Physics, vol. 51, no. 50, p. 503002, 2018.

[8] S. Spiga, A. Sebastian, D. Querlioz, and B. Rajendran, "Role of resistive memory devices in brain-inspired computing," in Memristive Devices for Brain-Inspired Computing, ser. Woodhead Publishing Series in Electronic and Optical Materials, S. Spiga, A. Sebastian, D. Querlioz, and B. Rajendran, Eds. Woodhead Publishing, 2020, pp. 3-16.

[9] N. Qiao, H. Mostafa, F. Corradi, M. Osswald, F. Stefanini, D. Sumislawska, and G. Indiveri, "A reconfigurable on-line learning spiking neuromorphic processor comprising 256 neurons and $128 \mathrm{k}$ synapses," Frontiers in neuroscience, vol. 9, p. 141, 2015.

[10] C. Frenkel, M. Lefebvre, J.-D. Legat, and D. Bol, "A 0.086-mm2 12.7pj/SOP 64k-synapse 256-neuron online-learning digital spiking neuromorphic processor in 28-nm CMOS," IEEE Transactions on Biomedical Circuits and Systems, vol. 13, no. 1, pp. 145-158, 2019.

[11] M. Payvand and G. Indiveri, "Spike-based plasticity circuits for alwayson on-line learning in neuromorphic systems," in 2019 IEEE International Symposium on Circuits and Systems (ISCAS). IEEE, 2019, pp. $1-5$.

[12] B. Widrow and M. Hoff, "Adaptive Switching Circuits," in 1960 IRE WESCON Convention Record, Part 4. New York: IRE, 1960, pp. 96-104. [Online]. Available: http://isl-www.stanford.edu/ $\sim\{\}$ widrow/papers/c1960adaptiveswitching.pd

[13] M. Payvand, Y. Demirag, T. Dalgaty, E. Vianello, and G. Indiveri, "Analog weight updates with compliance current modulation of binary rerams for on-chip learning," in 2020 IEEE International Symposium on Circuits and Systems (ISCAS). IEEE, 2020, pp. 1-5.

[14] W. Gerstner, M. Lehmann, V. Liakoni et al., "Eligibility traces and plasticity on behavioral time scales: experimental support of neohebbian three-factor learning rules," Front. Neur. Circ., vol. 12, p. 53, 2018.

[15] E. O. Neftci, "Data and power efficient intelligence with neuromorphic learning machines," iScience, vol. 5, pp. 52-68, 2018.

[16] M. Sanhueza and J. Lisman, "The camkii/nmdar complex as a molecular memory," Molecular brain, vol. 6, no. 1, pp. 1-8, 2013.

[17] G. Bellec, F. Scherr, A. Subramoney, E. Hajek, D. Salaj, R. Legenstein, and W. Maass, "A solution to the learning dilemma for recurrent networks of spiking neurons," bioRxiv, p. 738385, 2020.

[18] F. Zenke and S. Ganguli, "Superspike: Supervised learning in multilayer spiking neural networks," Neural computation, vol. 30, no. 6, pp. 1514 $1541,2018$.

[19] D. E. Rumelhart, G. E. Hintont, and R. J. Williams, "Learning representations by back-propagating errors," Nature, vol. 323, no. 6088, pp. 533-536, 1986.

[20] M. Davies, N. Srinivasa, T.-H. Lin, G. Chinya, Y. Cao, S. H. Choday, G. Dimou, P. Joshi, N. Imam, S. Jain et al., "Loihi: A neuromorphic manycore processor with on-chip learning," IEEE Micro, vol. 38, no. 1, pp. 82-99, 2018.

[21] S. Furber, F. Galluppi, S. Temple, and L. Plana, "The SpiNNaker project," Proceedings of the IEEE, vol. 102, no. 5, pp. 652-665, May 2014.

[22] A. Grübl, S. Billaudelle, B. Cramer, V. Karasenko, and J. Schemmel, "Verification and design methods for the brainscales neuromorphic hardware system," arXiv preprint arXiv:2003.11455, 2020.
[23] J. Backus, "Can programming be liberated from the von Neumann style?: a functional style and its algebra of programs," Communications of the ACM, vol. 21, no. 8, pp. 613-641, 1978. [Online]. Available: http://doi.acm.org/10.1145/359576.359579

[24] G. Indiveri and S.-C. Liu, "Memory and information processing in neuromorphic systems," Proceedings of the IEEE, vol. 103, no. 8, pp. 1379-1397, 2015.

[25] N. Qiao, C. Bartolozzi, and G. Indiveri, "An ultralow leakage synaptic scaling homeostatic plasticity circuit with configurable time scales up to 100 ks," IEEE Transactions on Biomedical Circuits and Systems, 2017.

[26] C. Bartolozzi and G. Indiveri, "Synaptic dynamics in analog VLSI," Neural Computation, vol. 19, no. 10, pp. 2581-2603, Oct 2007.

[27] M. Payvand, M. E. Fouda, F. Kurdahi, A. Eltawil, and E. O. Neftci, "Error-triggered three-factor learning dynamics for crossbar arrays," in 2020 2nd IEEE International Conference on Artificial Intelligence Circuits and Systems (AICAS). IEEE, 2020, pp. 218-222.

[28] A. Rubino, M. Payvand, and G. Indiveri, "Ultra-low power silicon neuron circuit for extreme-edge neuromorphic intelligence," in International Conference on Electronics, Circuits, and Systems, (ICECS), 2019, 11 2019, pp. 458-461.

[29] A. Payeur, J. Guerguiev, F. Zenke, B. A. Richards, and R. Naud, "Burstdependent synaptic plasticity can coordinate learning in hierarchical circuits," bioRxiv, 2020.

[30] D. Ielmini, S. Lavizzari, D. Sharma, and A. L. Lacaita, "Temperature acceleration of structural relaxation in amorphous ge2sb2te5," Applied Physics Letters, vol. 92, no. 19, p. 193511, 2008.

[31] M. Le Gallo, D. Krebs, F. Zipoli, M. Salinga, and A. Sebastian, "Collective structural relaxation in phase-change memory devices," Advanced Electronic Materials, vol. 4, no. 9, p. 1700627, 2018.

[32] I. Karpov, M. Mitra, D. Kau, G. Spadini, Y. Kryukov, and V. Karpov, "Fundamental drift of parameters in chalcogenide phase change memory," Journal of Applied Physics, vol. 102, no. 12, p. 124503, 2007.

[33] A. Pirovano, A. L. Lacaita, F. Pellizzer, S. A. Kostylev, A. Benvenuti, and R. Bez, "Low-field amorphous state resistance and threshold voltage drift in chalcogenide materials," IEEE Transactions on Electron Devices, vol. 51, no. 5, pp. 714-719, 2004.

[34] S. Kim, B. Lee, M. Asheghi, F. Hurkx, J. P. Reifenberg, K. E. Goodson, and H.-S. P. Wong, "Resistance and threshold switching voltage drift behavior in phase-change memory and their temperature dependence at microsecond time scales studied using a micro-thermal stage," IEEE Transactions on Electron Devices, vol. 58, no. 3, pp. 584-592, 2011.

[35] Y. Demirag, "Multiphysics modeling of Ge2Sb2Te5 based synaptic devices for brain inspired computing," Master's thesis, Ihsan Dogramaci Bilkent University, Ankara, Turkey, Jul. 2018.

[36] S. Nandakumar, M. Le Gallo, I. Boybat, B. Rajendran, A. Sebastian, and E. Eleftheriou, "A phase-change memory model for neuromorphic computing," Journal of Applied Physics, vol. 124, no. 15, p. 152135 , 2018.

[37] M. P. Lehmann, H. A. Xu, V. Liakoni, M. H. Herzog, W. Gerstner, and K. Preuschoff, "One-shot learning and behavioral eligibility traces in sequential decision making," Elife, vol. 8, p. e47463, 2019.

[38] M. Payvand, M. V. Nair, L. K. Müller, and G. Indiveri, "A neuromorphic systems approach to in-memory computing with non-ideal memristive devices: From mitigation to exploitation," Faraday Discussions, vol. 213, pp. 487-510, 2019.

[39] J. M. Brader, W. Senn, and S. Fusi, "Learning real-world stimuli in a neural network with spike-driven synaptic dynamics," Neural Computation, vol. 19, no. 11, pp. 2881-2912, 2007.

[40] T. Delbrueck and C. Mead, "Bump circuits," in Proceedings of International Joint Conference on Neural Networks, vol. 1, 1993, pp. 475-479.

[41] S.-C. Liu, J. Kramer, G. Indiveri, T. Delbruck, and R. Douglas, Analog VLSI:Circuits and Principles. MIT Press, 2002.

[42] I. Boybat, M. L. Gallo, T. Moraitis, T. Parnell, T. Tuma, B. Rajendran, Y. Leblebici, A. Sebastian, E. Eleftheriou et al., "Neuromorphic computing with multi-memristive synapses," Nature communications, vol. 9, p. $2514,2018$. 